\section{P3.332 MATCHING PARTICIPANTS IN A CHLAMYDIA SCREENING PROGRAMIME WITH PATIENTS SEEN BY STD CLINIC, GENERAL PRACTITIONER, OR SPECIALIST: ADDED VALUE OF A CHLAMYDIA SCREENING PROGRAMME}

doi:10.1136/sextrans-2013-051184.0785

1,2 G A F S van Liere, ${ }^{1,2} \mathrm{~N} \mathrm{H}$ T M Dukers-Muijrers, ${ }^{3,4}$ J E A M van Bergen, 5 V F van den Broek, ${ }^{6} \mathrm{H}$ M Götz, ${ }^{7} \mathrm{~J}$ S A Fennema, ${ }^{8} \mathrm{~F}$ S Stals, ${ }^{1,2} \mathrm{C}$ J P A Hoebe. 'Department of Sexual Health, Infectious Diseases and Environmental Health, Public Health Service South Limburg, Geleen, The Netherlands; 'Department of Medical Microbiology, Maastricht University Medical Center, Maastricht, The Netherlands; ${ }^{3}$ STI AIDS The Netherlands, Amsterdam, The Netherlands; ${ }^{4}$ Department of General Practice, Academic Medical Centre-University of Amsterdam, Amsterdam, The Netherlands; ${ }^{5}$ Epidemiology and Surveillance Unit, Centre for Infectious Disease Control, National Institute of Public Health and the Environment, Bilthoven, The Netherlands; ${ }^{6}$ Department of Infectious Disease Control, Rotterdam Rijnmond Public Health Service, Rotterdam, The Netherlands; ' ${ }^{7}$ luster of Infectious Diseases, Department of Research, Online Research and Prevention Unit, Amsterdam Health Service, Amsterdam, The Netherlands; ${ }^{8}$ Department of Medical Microbiology, Atrium Medical Centre, Heerlen, The Netherlands

Background Register and internet-based Chlamydia Screening Implementation (CSI) was introduced in the Netherlands to detect and treat asymptomatic infections and to limit ongoing transmission through annual testing and treatment of Chlamydia trachomatis $(\mathrm{Ct})$ in young people. This population-based screening may be compromised by addressing individuals who are already covered by regular care like general practitioners and STD clinics. We study, in a timeframe of five years, overlap between CSI participation and attendance of major services offering $\mathrm{Ct}$ screening services to assess whether CSI reached additional patients.

Methods Data included all Ct tests performed in subjects aged 16-29 years in Eastern South Limburg in the Netherlands (population 16-29 years: 42,000) between 2006 and 2010 by CSI, regional STD clinic, general practitioners (GPs), and specialists (mainly gynaecologists) as reported by the testing laboratory. Data were matched between testing agencies, with complete certainty (STD clinic) (name, date of birth) and with a certainty range (part of the name, month and year of birth). Maastricht University's ethical committee approved the study. Analyses were restricted to subjects tested (at least) by CSI.

Results In CSI, 4477 (82.9\%) subjects were first-time testers, others were previously tested by the STD clinic (3.5\%, $\mathrm{n}=190)$, GPs $(6.4 \%, \mathrm{n}=352)$, specialists $(3.6 \%, \mathrm{n}=197)$ or multiple providers $(3.4 \%, n=183)$. Compared to persons previously tested by the STD clinic/GPs/specialists, first time testers were younger (mean age $23 y$ vs. 24y, p < 0.001), and more often were heterosexual men compared to women $(29.8 \%$ vs. $13.6 \%, p<0.001)$. Ct prevalence was similar in first time testers (4.6\%) compared to previously tested persons $(4.9 \%, p=0.76)$. Symptom-rate was also similar $(2.5 \%$ vs. $3.3 \%$ $\mathrm{p}=0.32)$.

Conclusions Chlamydia screening addresses young individuals, including heterosexual men, hidden to current care with similar $\mathrm{Ct}$ prevalence and adds to the existing regular care.

\section{P3.333 CHLAMYDIA TRACHOMATIS IGG SEROPREVALENCE IN THE GENERAL POPULATION OF THE NETHERLANDS IN 1996 AND 2007}

doi:10.1136/sextrans-2013-051184.0786

${ }^{1} \mathbf{F}$ van Aar, ${ }^{1} \mathrm{M}$ de Moraes, ${ }^{2,3} \mathrm{~S}$ A Morré, $1,4,5 \mathrm{~J}$ E A M van Bergen, ${ }^{6} \mathrm{~F} \mathrm{R} \mathrm{M}$ van der Klis, ${ }^{7} \mathrm{~J}$ A Land, ${ }^{1,8} \mathrm{M}$ A B van der Sande, 'I V F van den Broek. 'Epidemiology \& Surveillance department, Center for Infectious Disease Control, National Institute for Public Health and the Environment, Bilthoven, The Netherlands; ' Laboratory of Immunogenetics, Department of Medical Microbiology and Infection Control, VU University Medical Center, Amsterdam, The Netherlands; IInstitute of Public Health Genomics, Department of Genetics and Cell Biology, Research Institutes CAPHRI and GROW, Faculty of Health, Medicine \& Life Sciences, University of Maastricht, Maastricht, The Netherlands;
${ }^{4}$ STI AIDS The Netherlands (SOA AIDS Nederland), Amsterdam, The Netherlands, ${ }^{5}$ Department of General Practice, AMC-UVA, Amsterdam, The Netherlands; 6 Laboratory for infectious disease and screening, Center for Infectious Disease Control, Epidemiology \& Surveillance department, Center for Infectious Disease Control, National Institute for Public Health and the Environment, Bilthoven, The Netherlands; ' ${ }^{7}$ Dept of Obstetrics and Gynaecology, University Medical Center Groningen, Groningen, The Netherlands; ${ }^{8}$ Julius Centre, UMCU, Utrecht, The Netherlands

Background Chlamydia trachomatis $(\mathrm{Ct})$ reporting rates from sexually transmitted infection (STI) clinics and general practitioners have shown a rising trend in the Netherlands. It is unknown to what extent this reflects increased $\mathrm{Ct}$ transmission or improved case-finding. To achieve more insight into the dynamics of the $\mathrm{Ct}$ epidemic, we explored the Ct IgG seroprevalence (marker of past Ct infection) in the general population of the Netherlands in 1996 and 2007.

Methods From two independent population-based studies in 1996 and 2007, serum samples were drawn, from 650 men and 1,000 women per study. Participants completed a questionnaire covering demographic information and sexual risk factors. Serum antibodies were analysed using Medac Ct IgG ELISA test. Multivariate logistic regression analyses explored changes in, and determinants of $\mathrm{Ct}$ IgG seroprevalence.

Results The Ct IgG seroprevalence was higher in women than in men in 1996 (11.3\% vs 5.2\%), but this difference had diminished in 2007 (8.4\% vs $6.9 \%)$. Among women aged $25-40$ years, the seroprevalence was significantly lower (OR 0.6 [0.4-0.8]) in 2007 than in 1996, whereas the seroprevalence among women aged 15-24 years and men in both age groups was slightly higher in 2007, though not significantly. Determinants of seropositivity were female gender (OR 1.7 [1.3-2.0]), known history of Ct infection (OR: 3.4 [1.7-6.8]), 25-40 years old (OR 1.6 [1.1-2.4]), non-western ethnicity (OR: 1.8 [1.1-3.2]) and $\geq 2$ recent sexual partners (OR: 2.2 [1.4-3.7]).

Conclusion Ct IgG seropositivity was associated with known STI risk factors. Our results suggest that the proportion of individuals in the population who have had a $\mathrm{Ct}$ infection did not increase between 1996 and 2007. The decrease in seroprevalence among women aged 25-40 years between 1996 and 2007 may indicate changes in Ct transmission due to a more pro-active 'test and treat' policy.

\section{P3.334 SOCIAL GEOGRAPHY OF DIAGNOSED AND UNDIAGNOSED STIS: PRELIMINARY RESULTS}

doi:10.1136/sextrans-2013-051184.0787

${ }^{1}$ C F Turner, ${ }^{2} \mathrm{~S} M$ Rogers, ${ }^{3} \mathrm{~J}$ R Chromy, ${ }^{4} \mathrm{~A}$ M Roman, ${ }^{5} \mathrm{~W}$ C Miller, ${ }^{2} \mathrm{~S}$ Tan. ${ }^{1}$ City University of New York, New York, NY, United States; ${ }^{2}$ Research Triangle Institute, Washington, DC, United States; ${ }^{3}$ Research Triangle Institute, Research Triangle Park, NC, United States; ${ }^{4}$ University of Massachusetts, Boston, MA, United States; ${ }^{5}$ School of Medicine, University of North Carolina, Chapel Hill, NC, United States

Background Geographic propinquity affects the number and types of sexual partners available as well as sexual network connexions. Geographic location also affects the pervasiveness of STI screening and the availability of STI treatment. This presentation explores the geographic clustering of diagnosed and undiagnosed STIs and STI-risk factors in CityplaceBaltimore, StateMD.

Methods The 2006-2009 Monitoring STIs Survey Program (MSSP) tracked trends in three STIs (trichomoniasis, chlamydia, gonorrhoea) in probability samples of the population ages 15 to 35 in CityplaceBaltimore, StateMD. 2,936 participants reported on previously diagnosed STIs and STI-risk behaviours, and 2,136 participants provided biospecimens for STI testing. Census tract codes and socio-demographic characteristics of these tracts were appended to the MSSP data. Investigators are exploring the social geography of the distribution of diagnosed and undiagnosed STIs and STI-risk factors using this database. 